\title{
La participation comme objet intermédiaire d'apprentissages: leçons d'une démarche participative sur les pollutions diffuses agricoles
}

\author{
Laura Seguin $^{1, *}$ (D, Fabienne Barataud ${ }^{2}$ (D) Laurence Guichard ${ }^{3}$, Mathilde Bonifazi ${ }^{3}$, \\ Véronique Souchère $^{4}$ (D), Sami Bouarfa ${ }^{5}$ (D) et Julien Tournebize ${ }^{6}$ (D) \\ 1 Sociologie, INRAE, UMR G-EAU, Montpellier, France \\ ${ }^{2}$ Géographie, INRAE, UE ASTER, Mirecourt, France \\ ${ }^{3}$ Agronomie, INRAE, AgroParisTech, UMR Agronomie, Thiverval-Grignon, France \\ ${ }^{4}$ Agronomie, INRAE, AgroParisTech, UMR SADAPT, Thiverval-Grignon, France \\ ${ }^{5}$ Sciences de l'eau, INRAE, UMR G-EAU, Montpellier, France \\ ${ }^{6}$ Hydrologie, INRAE, UR HYCAR, Antony, France
}

Reçu le 18 juillet 2019. Accepté le 16 mars 2021

L'indispensable changement des pratiques agricoles pour lutter contre la pollution des eaux est inscrit à l'agenda public depuis des décennies, sans beaucoup de résultats. Des initiatives locales originales et prometteuses continuent toutefois à ouvrir des perspectives. NSS s'en était déjà fait l'écho, notamment en $2015(23,1)$ avec l'article de M. Gisclard et al. C'est également le cas de la démarche participative qu'analysent les auteurs de cet article. Ils le font de manière originale, sous l'angle des apprentissages opérés à l'occasion de la co-ingénierie d'un projet de recherche entre chercheurs et acteurs, puis lors de sa mise en œuvre locale avec des acteurs agricoles et non agricoles. Mobilisant la notion de cadrage, ils montrent comment la participation permet de transformer les cadrages cognitifs et politiques initiaux du projet de recherche, et parvient à rendre discutable le problème de la pollution agricole de l'eau. Les effets apparaissent toutefois moins marqués lors de la mise en œuvre du projet, au moins à court terme, du fait de la perpétuation des rapports inégaux entre acteurs agricoles et acteurs de l'eau.

La Rédaction

\begin{abstract}
Résumé - Cet article propose d'analyser la manière dont la construction transdisciplinaire d'un projet de recherche, puis la mise en œuvre d'une démarche participative sur l'enjeu des pollutions diffuses agricoles de l'eau (nitrates et pesticides), constituent des espaces d'apprentissages. L'étude s'appuie sur une recherche participative menée sur le territoire de la Brie, en Seine-et-Marne, analysée en tant que processus permettant de transgresser les frontières disciplinaires, qui opposent de manière souvent arbitraire chercheurs et acteurs, et les frontières qui divisent les différentes parties prenantes d'un problème public. Les perceptions et les connaissances initiales des chercheurs et acteurs s'enrichissent à travers la participation. Les limites de ce processus et des inégalités d'apprentissages sont également identifiées.
\end{abstract}

Mots-clés : agriculture / environnement / transdisciplinarité / participation / apprentissage / gestion de l'eau

\begin{abstract}
Participation as intermediary object of learning: lessons from a participatory approach to agricultural non-point source pollutions. This article analyzes learning processes resulting from the collective construction of a transdisciplinary research project, and from the implementation of a participatory approach to agricultural non-point source pollutions (nitrates and pesticides). The study is based on a participatory research project carried out in the Brie plain of Seine-etMarne (France). It is regarded as a border-crossing process across disciplinary boundaries which often
\end{abstract}

\footnotetext{
*Auteur correspondant : laura.seguin@inrae.fr
} 
arbitrarily oppose researchers and stakeholders, as well as dividing the different stakeholders concerned by a public issue. The analysis first examines learnings resulting from a preliminary stage of participation coengineering that involve scientists and their socio-professional partners, then learnings resulting from the participatory process with the local actors. The results show that the researchers and actors learn to shift their gaze from a technical object to a political one, linked to a rising awareness of the level of conflictuality which the participatory approach might assume. They also acquire new knowledge from the participating disciplines and from the local actors, as well as participatory skills. These learning processes may change everyone's perceptions about the issue addressed, the territory and the actors involved. However, the study also revealed the limitations and learning assymetries of the process.

Keywords: farming / environment / transdisciplinarity / participation / learning process / water management

Sur les questions environnementales, l'heure est à la recherche interdisciplinaire, voire transdisciplinaire ${ }^{1}$, conduite en partenariat entre chercheurs et acteurs socioprofessionnels, et de manière participative. À ces nouvelles approches sont attribués plusieurs types de vertus. L'intégration disciplinaire entre sciences de la nature et sciences sociales permet de traiter des phénomènes complexes à la frontière entre nature et société. Les questions environnementales font naître un besoin de connaissance «à la jonction du scientifique et du sociétal» (Jollivet et Legay, 2005) auquel la transdisciplinarité permet de répondre. Les recherches construites «en partenariat» avec des acteurs socioprofessionnels favorisent le développement de travaux «socialement utiles et utilisables », développés à partir de questions pratiques posées par ces derniers, et dont ils sont capables de tirer parti une fois la recherche terminée (Soulard et al., 2007). Enfin, cela répond à un enjeu démocratique d'inclusion du plus grand nombre dans la construction des savoirs et des décisions en matière d'environnement, et à un enjeu plus pragmatique de meilleure implication des acteurs dans la mise en œuvre d'actions décidées «en concertation». Ouvrir le cercle des « sachants » et des décideurs à une pluralité d'acteurs, au-delà de ceux habituellement habilités à définir et à qualifier les enjeux environnementaux (Barbier et Larrue, 2011, p.93), contribue à améliorer la qualité des décisions dans ce domaine (Reed, 2008).

Au cœur de ces interrelations multiples se niche une dimension pédagogique que les analyses n'explicitent pas toujours. Chercheurs de disciplines différentes, partenaires institutionnels ou socioprofessionnels,

\footnotetext{
1 Nous entendons par interdisciplinarité un dialogue enrichissant entre plusieurs disciplines, la transdisciplinarité, elle, consiste à faire coopérer des chercheurs de différentes disciplines et des non-chercheurs évoluant à la marge ou en dehors de la recherche, et à intégrer ces diverses formes de savoirs : «La transdisciplinarité s'impose dès lors qu'un sujet nourrit des incertitudes et des interrogations qui vont interpeller et faire coopérer tant des chercheurs de disciplines diverses que des acteurs évoluant en dehors des sphères de la recherche» (Raymond, 2017, p. 42).
}

acteurs et citoyens dont les savoirs, les intérêts et les représentations de l'enjeu diffèrent, s'engagent pour faire valoir leurs savoirs et leurs points de vue, orienter ou transformer l'objet discuté «et se trouvent transformés en retour ${ }^{2} »$. Cet article propose, à partir d'une recherche ancrée dans ce renouvellement des manières de faire, d'examiner les apprentissages qu'elle occasionne pour ceux y prenant part. Les apprentissages sont l'un des effets identifiés par les recherches sur la participation (Blondiaux et Fourniau, 2011). Nous les entendons comme processus de (trans)formation des acteurs, de leurs savoirs et savoir-faire, et parfois de leurs représentions et attitudes (Seguin, 2020). Quels sont les apprentissages effectués et jusqu'à quel point les acteurs apprennent-ils? Jusqu'où la participation permet-elle de «transgresser les frontières » (Billaud, 2003, p. 29), à la fois disciplinaires, mais aussi celles qui opposent - de manière souvent arbitraire - chercheurs et acteurs, et celles qui divisent enfin les différentes parties prenantes d'un problème public? Quelles sont les potentielles limites et asymétries d'apprentissages?

Le projet Brie'eau ${ }^{3}$, objet de cet article, porte sur l'enjeu de la qualité de l'eau et des pollutions diffuses agricoles dans la Brie, région de grandes cultures en Seine-et-Marne. Dans un premier temps, nous donnons quelques éléments de définition, de contexte et de méthodologie. Puis nous montrons comment le processus de «co-ingénierie» de la participation constitue un premier espace d'apprentissages pour les scientifiques et les partenaires du projet, à la fois entre divers mondes disciplinaires, et entre chercheurs et acteurs socioprofessionnels de l'eau et des questions agricoles. Enfin, dans la dernière partie, nous examinons un second espace

\footnotetext{
Expression de Sandrine Rui (2004) concernant des dispositifs participatifs sur des projets d'aménagement: «Les participants s'engagent dans la discussion pour transformer le projet et se voient transformés en retour».

3 Projet financé par le programme Pour et sur le développement régional (PSDR) de la région Île-de-France et le PIRENSeine.
} 
d'apprentissages correspondant à la mise en œuvre du processus participatif auprès des acteurs locaux.

\section{Enquêter sur les apprentissages d'une recherche participative}

\section{Expériences participatives et apprentissages}

Les travaux sur les dispositifs de participation identifient plusieurs types d'apprentissages. Les apprentissages cognitifs concernent l'appropriation de savoirs, qu'ils soient experts, professionnels ou d'usage (Nez, 2011). Les apprentissages politiques désignent l'acquisition de compétences, de savoir-faire ou d'aptitudes favorisant la participation à une discussion ou une à action collective : capacités à prendre la parole, écouter, argumenter, monter en généralité, animer une discussion, gérer une négociation conflictuelle, etc. (Talpin, 2011; Gardesse, 2011). Les apprentissages organisationnels désignent la construction de nouvelles formes d'organisation et/ou de discussion entre acteurs (Barnett, 2001 ; Simard et Fourniau, 2007). Ces trois formes d'apprentissages sont généralement étudiées conjointement (Daré et al., 2010).

Les travaux s'intéressent aussi bien aux commanditaires et concepteurs des dispositifs participatifs (élus, acteurs institutionnels, chercheurs, etc.) qu'aux participants mobilisés (acteurs socioprofessionnels ou associatifs, citoyens/habitants ${ }^{4}$ ). Que les apprentissages soient individuels ou collectifs, ils relèvent d'un processus fait d'interactions sociales, et peuvent être qualifiés d'apprentissage social [social learning] (Webler et al., 1995; Reed et al., 2010).

La littérature scientifique montre cependant que la participation n'a que peu d'effets sur les individus en matière de changement d'opinion. Julien Talpin (2011, p.152) remarque, par exemple, que les préférences individuelles ne changent pas chez les participants aux budgets participatifs qu'il observe, du fait qu'elles se sont formées au cours de socialisations antérieures, et donc stables et solides. C'est également ce que montrent les travaux sur les apprentissages dans l'analyse des politiques publiques: ceux-ci ne portent généralement que sur des dimensions secondaires et non sur les systèmes de croyance ou "visions du monde» propres aux acteurs (Sabatier et Jenkins-Smith, 1993). Mais c'est que dans ces processus de changement institutionnel ou

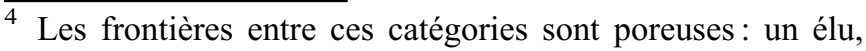
acteur institutionnel, socioprofessionnel ou associatif pouvant être tantôt commanditaire d'un dispositif, tantôt participant. En revanche, les citoyens ou habitants sont plus rarement à l'origine ou concepteurs de dispositifs participatifs.
}

de réforme administrative, la dimension participative n'est, justement, pas toujours pensée pour favoriser « la construction de significations communes » (Lascoumes, 2010, p. 636). Lorsque les interactions entre acteurs sont en revanche organisées dans cette perspective, mobilisant des outils de dialogue spécifiques pour l'échange de savoirs et la délibération collective, les effets d'apprentissages peuvent se révéler plus profonds: «Des échanges, sous la forme de débats dans des forums spécialisés, peuvent favoriser des changements progressifs (incrémentaux) dans les systèmes de croyance » (Genieys et Hassenteufel, 2012, p. 94). Il s'agit donc de porter une attention spécifique aux mises en situation d'apprentissages des acteurs permises par certains dispositifs. Pour les analyser, nous mobilisons la notion de «cadrage» au sens de framing (Goffman, 1974), c'est-à-dire le travail de construction de sens de l'action collective. Cette notion s'applique ici à l'analyse d'un projet de recherche dont l'objet discuté est soumis à un certain cadrage: problème et solutions mis en débat et public constitué (cadrage sociopolitique); types de savoirs ou connaissances mobilisés et/ou construits (cadrage cognitif). Dans ces opérations de cadrage, les choix effectués peuvent jouer un rôle non négligeable sur la nature et la portée des apprentissages.

\section{Une recherche initialement construite autour d'une innovation}

Dans la Brie, l'activité agricole de type grandes cultures a conduit à une forte dégradation de la qualité de l'eau (contamination par les nitrates et produits phytosanitaires) de la nappe des calcaires de Champigny, principale ressource en eau souterraine d'Île-de-France. Dès 2005, des rencontres ont lieu entre des chercheurs en hydrologie et biologie des écosystèmes de l'IRSTEA ${ }^{5}$ (UR HYCAR, Antony) et AQUI Brie, association de connaissance et de protection de la nappe de Champigny. Sur un sous-bassin versant de l'Ancœur, où l'association mène une animation agricole tournée vers des pratiques plus économes en intrants, une première expérimentation d'implantation de zones tampons humides artificielles (ZTHA) est menée avec les chercheurs. Les ZTHA sont une solution d'ingénierie écologique permettant de récupérer temporairement les eaux de drainage agricole avant qu'elles ne rejoignent la nappe et de retenir une partie des contaminants qu'elles contiennent grâce à un phénomène d'épuration naturelle [absorption, dégradation des molécules] (Tournebize et al., 2017). Sans

\footnotetext{
${ }^{5}$ Institut national de recherche en sciences et technologies pour l'environnement et l'agriculture, fusionné début 2020 avec l'INRA pour devenir INRAE (Institut national de recherche pour l'agriculture, l'alimentation et l'environnement).
} 
revenir ici sur cette première expérimentation, notons qu'elle a été analysée comme un processus d'innovation « co-construit orienté» (Tournebize et al., 2012) avec les acteurs locaux, et notamment avec un petit groupe d'agriculteurs. Le dialogue avec ces derniers n'avait pas alors été pensé comme un processus de construction collective.

Débutant en 2016, le projet Brie'eau s'inscrit dans la continuité de cette première expérience tout en intégrant de nouvelles questions de recherche et une dimension interdisciplinaire (hydrologie, agronomie, écologie, droit et sociologie). Il propose de poursuivre les recherches sur l'efficacité des ZTHA en termes d'abattement des polluants contenus dans les eaux agricoles. Il ouvre la réflexion sur un autre service écosystémique rendu par ces aménagements : la biodiversité. Il intègre une étude des possibilités de reconnaissance réglementaire des ZTHA et propose enfin la conception, la mise en œuvre et l'évaluation d'un processus de dialogue territorial. Des professionnels et consultants (Lisode, bureau d'étude spécialisé dans l'accompagnement de la concertation et Biotope, bureau d'étude naturaliste) sont sollicités pour certaines compétences en appui aux chercheurs. L'ambition du projet est aussi de se situer à une échelle territoriale supérieure, celle du bassin versant, et d'intégrer un panel d'acteurs locaux plus diversifié. Le projet est conduit en partenariat avec des acteurs socioprofessionnels du territoire concerné : AQUI Brie, la chambre d'agriculture et la direction départementale des territoires de Seine-et-Marne. Ces chercheurs et acteurs sont désignés par «groupe pilote» du projet.

\section{Identifier les apprentissages à deux niveaux}

Afin de mener l'enquête sur les processus d'apprentissages, un double protocole méthodologique a été mis en œuvre. Le premier est centré sur le groupe pilote. Initialement mobilisés pour un domaine d'expertise propre ou le portage d'outils spécifiques, les membres de ce groupe ont progressivement été impliqués dans la conception et la conduite de la démarche participative via un travail collectif sur son cadrage politique et cognitif. L'enquête conjugue observation participante des différents moments de ce travail et entretiens semi-directifs menés au début puis à la fin du projet. S'agissant d'identifier des processus de changement, il est en effet nécessaire de saisir un état initial des savoirs et des perceptions des acteurs, de constater le cheminement collectif effectué au fil du temps par l'observation, et de faire ensuite un état des lieux final selon une même grille d'analyse.

Le second protocole concerne les acteurs mobilisés dans la démarche participative. Il s'agit d'agriculteurs, d'acteurs des filières agricoles (coopératives, négoces), d'industriels, d'acteurs institutionnels, de représentants de collectivités en charge de l'eau potable, d'associations environnementales et d'usagers du territoire. Ces acteurs ont été réunis six demi-journées entre 2017 et 2019 sous forme d'ateliers ou de rencontres sur le terrain, mobilisant à chaque fois une trentaine de participants (Fig. 1). Les différentes étapes avaient plusieurs objectifs: d'abord permettre l'expression des perceptions, valeurs, objectifs et contraintes (notamment professionnelles) respectives; ensuite échanger les savoirs entre scientifiques et acteurs locaux; puis se projeter et imaginer des changements à travers la construction de scénarios agronomiques du territoire; et enfin stimuler des discussions et des négociations sur des actions individuelles et collectives à mettre en œuvre localement grâce à un jeu de rôle. Une séance de restitution a permis de faire la synthèse du travail accompli et d'envisager les suites de cette démarche ${ }^{6}$.

Pour identifier les apprentissages issus de ce processus, les différents moments ont fait l'objet d'observations, d'un enregistrement et d'une retranscription des échanges. Des débriefings collectifs après chaque rencontre étaient spécifiquement orientés vers l'identification des enseignements de la rencontre, des surprises, étonnements ou encore des frustrations ou gênes ressentis au cours de la participation. Le débriefing est un moment crucial d'apprentissage, en particulier à l'issue d'un jeu de rôle (Crookall, 2014). Ces temps d'échanges, en petits groupes puis en groupe entier, étaient animés par des observateurs extérieurs qui revenaient sur les points saillants des échanges, les productions des groupes, et qui encourageaient la réflexivité des participants et l'autoévaluation de leurs acquis. Enfin, des entretiens ont été conduits avec une partie des participants en amont et à l'issue de l'expérience.

\section{Une «co-ingénierie » de la participation source d'apprentissages}

La co-ingénierie de la participation (Daniell et al., 2010) est un processus au cours duquel un groupe d'acteurs décide du cadrage politique et cognitif d'une démarche participative. Ce travail contribue à faire émerger des différences de point de vue entre acteurs pilotes de la participation, un «débat sur le débat» (Fourniau, 2007) qui nécessite de s'accorder. Ce processus demande un investissement important mais permet de gagner ensuite en efficacité, en diminuant les risques de remise en cause des objectifs et des méthodes en cours de route. Ainsi, le groupe pilote du projet Brie'eau s'est réuni près d'une quinzaine de fois courant 2017 et 2018.

${ }^{6}$ Pour plus de détails sur le déroulement de cette démarche voir (Seguin et al., 2018). 


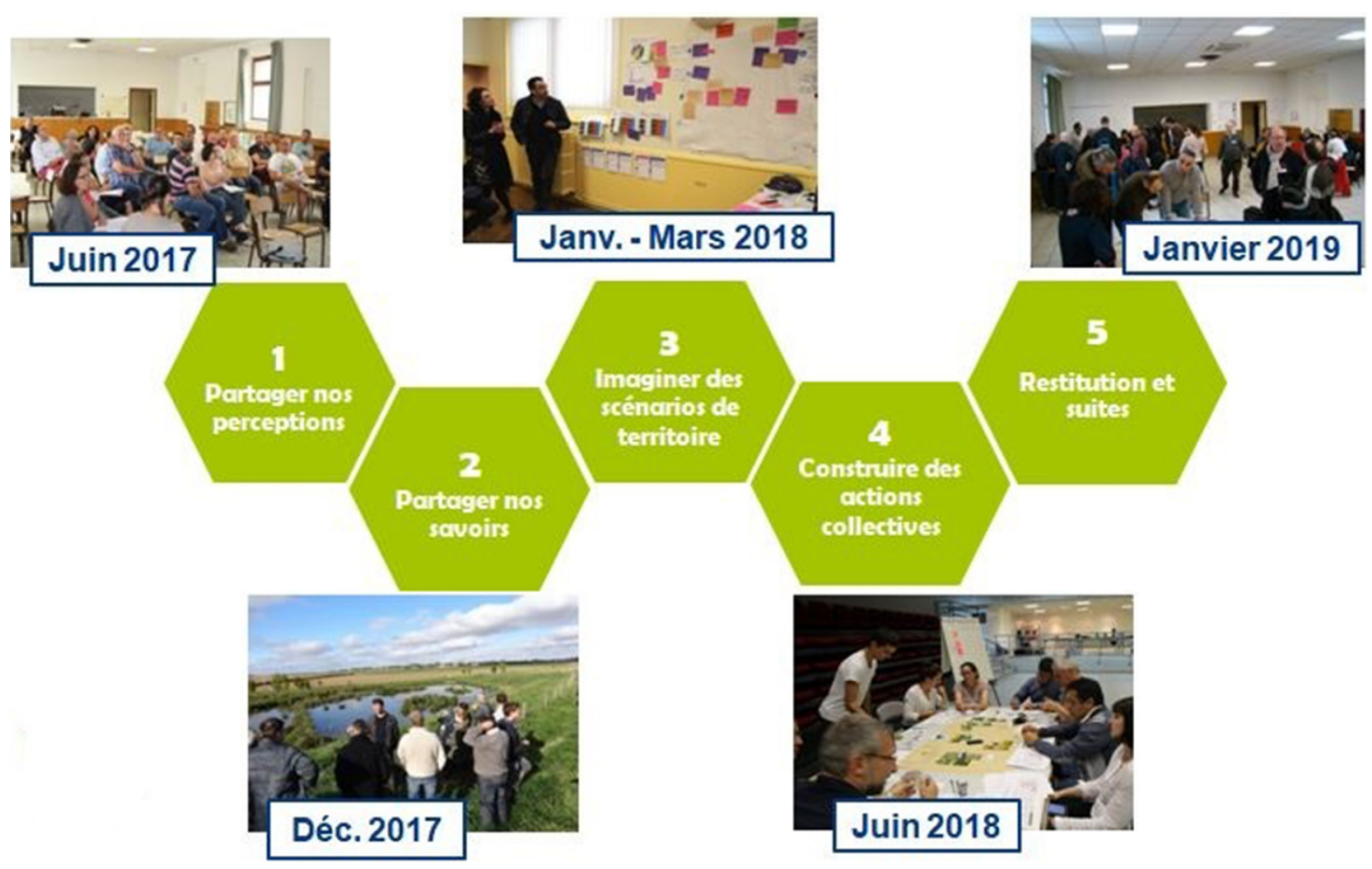

Fig. 1. Schéma et illustrations de la démarche participative (@ INRAE, Julien Tournebize, Laura Seguin).

\section{Du technique au sociopolitique: rendre discutable un problème public}

Le cadrage initial du projet Brie'eau place les ZTHA au cœur de la démarche participative. Il s'agit à l'origine d'étudier les «modalités de transfert d'une innovation» vers les usagers des espaces agricoles - principalement les agriculteurs. Ainsi, le premier outil mobilisé, Mete'eau (Barataud et al., 2015), est repensé dans le cadre de ce projet pour intégrer de nouveaux objets ou thématiques («zones tampons», «biodiversité», modalités de transferts hydriques en zone drainée...). Cette adaptation, en obligeant à expliciter les termes, les enjeux et les manières de représenter, constitue une première médiation entre chercheurs et acteurs partenaires. Elle fait notamment naître des débats sur le type de zone tampon à intégrer à la réflexion: s'agit-il uniquement des ZTHA ou bien ouvre-t-on la discussion à une plus grande diversité de zones tampons (bande enherbée, haie, ripisylve)? Un premier recadrage de l'objet est donc observable suite à la reconnaissance que le groupe pilote n'en a pas une conception homogène et partagée.

Ce processus se poursuit au cours de plusieurs rencontres visant à remettre à plat les objectifs de la participation. Deux séances en particulier, dont l'animation est construite à partir de la méthode ARDI (Acteurs, Ressources, Dynamiques et Interactions) développée dans le domaine de la modélisation d'accompagnement (Étienne et al., 2011), y sont consacrées. Il s'agit d'abord de redéfinir la question de départ au cœur de la démarche participative. Au fil des échanges se profile un recadrage de l'objet, passant d'une entrée par les solutions techniques (zones tampons) à une entrée par le problème des pollutions diffuses agricoles. Le groupe construit ainsi un positionnement collectif différent de la philosophie initiale du projet. Il s'accorde sur le fait que la démarche participative ne vise pas à déployer une innovation technique par la construction de son acceptabilité sociale, mais bien à ouvrir le débat sur les pollutions diffuses, en mettant en discussion deux leviers d'action complémentaires : les zones tampons et les changements de pratiques agricoles. Ces deux leviers sont proposés comme des alternatives aux pratiques actuelles qui doivent également être représentées et discutées dans la démarche. Celle-ci est alors envisagée comme un processus donnant l'occasion de «rendre discutables ${ }^{7} \gg$ les questions d'agriculture sur le territoire et d'évaluer la pertinence de diverses solutions. Une représentante d'AQUI Brie relate ainsi la manière dont elle a perçu cette évolution :

«Au départ, il y avait une confusion sur les objectifs entre la stratégie de déploiement des zones tampons... le projet Brie'eau devait contribuer vraiment à déployer les zones tampons et à la fin du projet, on saurait où implanter

\footnotetext{
7 Nous empruntons l'expression à Yannick Barthe (2002), penseur d'une «démocratie technique» permettant de rendre discutables, et donc révisables, les choix technico-scientifiques effectués par le passé.
} 
des zones tampons, à tels endroits, et qu'on aurait vraiment des objectifs très opérationnels [...]. Et finalement la formulation, après, des objectifs des ateliers, c'était un projet de dialogue autour des pollutions, de la lutte contre les pollutions diffuses et du changement des pratiques agricoles ».

Ce déplacement a pu rencontrer des résistances. Le représentant de la chambre d'agriculture reconnaît ses difficultés à «accepter le fait qu'on élargisse le débat » à la question des pratiques agricoles. Lors d'un séminaire de bilan, il évoque pourtant un enseignement majeur du projet: gestion des pressions (par les pratiques agricoles) et gestion des transferts (par les zones tampons) «doivent être liées pour traiter des problèmes de pollutions diffuses », ce qui contraste avec sa conception initiale du cadrage du débat.

La question du public est également un élément du cadrage politique de la démarche. Initialement, ceux pour qui la participation est un outil de déploiement des zones tampons considèrent que les acteurs à mobiliser - les propriétaires fonciers (publics, privés, agriculteurs, etc.) et les potentiels financeurs - sont ceux à même de jouer un rôle opérationnel. En revanche, ceux favorables à un dialogue sur les pollutions diffuses souhaitent l'ouvrir à un public large incluant les acteurs agricoles (agriculteurs et filières), les acteurs de l'eau potable, mais aussi d'autres usagers du territoire et jusqu'aux citoyens généralement tenus éloignés de ces questions. La méthode ARDI permet de mettre en évidence ces dissensions jusqu'alors implicites et joue ce rôle d'intermédiation permettant finalement de s'accorder. Une fois clarifié l'objet de la participation, il est admis que les questions d'eau et d'agriculture concernent une diversité d'acteurs. Le groupe pilote choisit d'ouvrir la démarche à des parties prenantes d'horizons divers pour faire se rencontrer monde agricole et monde de l'eau, mais aussi impliquer d'autres usagers du territoire sous forme d'acteurs organisés (fédération de chasse, association environnementale). Cependant, le choix est fait de ne pas mobiliser citoyens et habitants du territoire (ni professionnels ni acteurs organisés). Cette question est débattue à plusieurs reprises mais d'importantes réticences subsistent face aux risques politiques perçus si les pollutions diffuses venaient à émerger comme un problème public local au-delà du dispositif de recherche. Le passage d'un objet technique à un objet politique s'accompagne d'une prise de conscience du degré de conflictualité que le dispositif pourrait endosser. Le choix de ne mobiliser que des associations (d'usagers, environnementales) s'explique par un souci d'ouverture maîtrisée, avec des acteurs habitués au dialogue. De même, les agriculteurs mobilisés par l'association AQUI Brie sont majoritairement sensibles aux questions de qualité d'eau (sans toutefois être tous engagés dans des pratiques économes en intrants) et ouverts à la discussion, malgré leur méfiance en début de procédure ${ }^{8}$. À l'issue de l'expérience, on reconnaît l'intérêt de cette première ouverture (même si elle reste relative), comme l'exprime un représentant d'AQUI Brie :

«Un des apprentissages intéressants, c'est que d'autres publics peuvent s'intéresser à cette question-là, des publics qui sont complètement... étrangers à ces questions. Et que le public agricole n'est pas finalement hostile à mettre ces questions-là sur la table».

\section{La construction d'un regard transdisciplinaire}

Le projet Brie'eau est assez révélateur de l'évolution des recherches environnementales, en particulier celles portant sur la gestion de l'eau: bien que la demande de science reste principalement adressée aux hydrologues et aux agronomes, les chercheurs en sciences sociales s'y invitent de plus en plus régulièrement (Barataud et al., 2017). À l'origine, c'est principalement parce qu'ils sont perçus comme ayant une «fonction d'aide à la prise de recul et de médiation » (Barataud et al., 2017, p. 6). Mais nous voyons combien ils contribuent finalement à redéfinir le problème et la manière de le rendre discutable, venant en cela nourrir le processus de coingénierie de la participation.

La première phase du projet Brie'eau consiste à construire une nouvelle forme de connaissance à travers une analyse des représentations et des valeurs attachées au territoire. Cette approche compréhensive est menée grâce à des entretiens mettant en évidence la diversité des perceptions des acteurs locaux sur différents objets du territoire: loin d'être appréciée uniquement en fonction de son efficacité technique, l'innovation que constitue la ZTHA fait l'objet d'une évaluation suivant des logiques économiques, sociales ou politiques, voire recouvre des enjeux symboliques forts pour les différents acteurs (Seguin et Barataud, 2021). Les entretiens contredisent également l'une des hypothèses de départ qui consistait à considérer la biodiversité comme un enjeu plus fédérateur et moins conflictuel que celui de la qualité de l'eau. Certains membres du groupe pilote prennent conscience qu'il s'agit d'un enjeu suscitant tout autant de distance critique, à la fois chez les agriculteurs et chez certains représentants de collectivités: «Je ne m'attendais pas à ce que la connaissance et l'appétence vis-à-vis des questions de biodiversité soient si faibles » (représentant AQUI Brie). C'est donc une autre connaissance du territoire qui est

\footnotetext{
${ }^{8}$ La participation des agriculteurs reposait sur une démarche volontaire, après invitation par courrier, téléphone et mail des agriculteurs du bassin versant. Ils sont dans leur grande majorité en agriculture conventionnelle, une minorité est engagée dans des pratiques de réduction d'intrants.
} 
progressivement intégrée à celle des hydrologues et des agronomes.

La construction d'un regard transdisciplinaire se poursuit avec la démarche Co-Click'eau (Gisclard et al., 2015) qui intervient dans la phase de construction de scénarios de territoire. C'est au cours de l'adaptation de cette démarche aux objectifs du projet que savoirs disciplinaires et savoirs des acteurs partenaires circulent. Les données intégrées dans l'outil ainsi que les scénarios de territoire produits constituent des objets intermédiaires à travers lesquels connaissances, objectifs et intérêts des acteurs sur le problème perçu comme sur ses résolutions possibles s'expriment. Modéliser les surfaces de cultures nécessite de partager des données locales agronomiques issues d'expertises précédentes - notamment celles de la chambre d'agriculture - afin de représenter le plus fidèlement possible l'assolement du territoire et les différentes stratégies de conduite des cultures. Surtout, cela nécessite d'y intégrer des éléments prospectifs permettant d'imaginer des changements, par exemple le développement d'une nouvelle culture ou d'une stratégie culturale innovante. Cet exercice s'accompagne pour certains d'une acquisition de nouvelles connaissances concernant les leviers agronomiques permettant de réduire les intrants. En parallèle, afin d'intégrer dans l'outil des paramètres tels que les types de sols et les différents mécanismes hydrologiques du territoire, il est nécessaire de partager un même niveau de connaissances et le même vocabulaire. Le groupe s'approprie des savoirs en hydrologie utiles pour comprendre la complexité des processus de transfert des polluants. Support de la construction d'une expertise collective mêlant connaissances scientifiques et professionnelles, l'outil Co-click'eau a donc joué le rôle d'objet intermédiaire pour la construction d'une transdisciplinarité au sein de ce groupe pilote de chercheurs et d'acteurs.

\section{Repenser le rôle de l'expertise en situation de participation}

L'expérience a contribué à construire, chez les chercheurs et les partenaires porteurs d'une forme d'expertise, une posture nouvelle vis-à-vis de la mobilisation de savoirs scientifiques et techniques en situation de débat public. Jusqu'alors, la démarche Coclick'eau avait été mise en œuvre dans le cadre d'aires d'alimentation de captage à destination d'acteurs disposant de compétences techniques dans le domaine agronomique, ou avec des gestionnaires ou animateurs de territoire. Le constat était fait d'une plus grande facilité de mise en œuvre dans des arènes homogènes comme des comités techniques agricoles. Brie'eau propose un autre cadrage de l'outil destiné à un public plus hétérogène intégrant des individus profanes des questions agricoles (représentants de collectivités, acteurs associatifs). Ce défi place les membres du groupe pilote face à des choix d'usage et d'animation de l'outil différant des pratiques antérieures. Alors que certains dans le groupe mettent l'accent sur la précision de l'expertise et des données techniques à présenter, d'autres souhaitent leur simplification pour en faciliter l'appropriation par un public hétérogène. Lors du premier atelier construit avec Co-click'eau, l'expertise occupe une place centrale, conduisant à limiter les échanges entre acteurs. Placés devant des indicateurs jusque-là inconnus, les participants éprouvent des difficultés d'appropriation et en réfèrent aux experts présents. Tenant compte de ces difficultés, le groupe pilote choisit ensuite de reconfigurer la place de l'expertise dans l'animation du second atelier. Il décide de privilégier des données et des indicateurs simplifiés et agrégés à l'échelle du territoire pour en faciliter la lecture et l'appropriation. Une agronome reconnaît que cet exercice a été riche d'enseignements :

«Ce n'est pas forcément facile à accepter pour des chercheurs qui souhaiteraient d'abord dévoiler toutes les données pour ensuite permettre la discussion. Là, on ne montrait pas tout, on ne montrait que des données agrégées, avec une crainte que ça n'allait pas fonctionner [...]. C'est super révélateur pour nous, c'est qu'on se plante à penser qu'il faut vraiment tout expliquer par le menu, dans le détail, pour faire discuter les gens. Alors qu'à ce grain-là, ça permet déjà d'enclencher des mouvements, des discussions, des échanges, d'autres points de vue».

Ce déplacement place les scientifiques et leurs partenaires dans une situation d'inconfort, où le risque identifié est d'en dire peu et donc de manquer de précision. Cependant, ils en reconnaissent les bénéfices en termes de débat public puisque, dans une logique pédagogique, l'expertise scientifique et technique simplifiée s'articule plus facilement aux savoirs d'usage et aux savoirs professionnels des participants ainsi qu'à leurs propres questionnements.

\section{L’acquisition de savoir-faire participatifs}

Enfin, le travail de co-ingénierie engage une réflexion sur l'animation des ateliers : permettre l'écoute active et l'expression écrite et orale, prendre en compte les inégalités des participants devant la prise de parole publique en diversifiant les supports d'expression et les modalités de travail (en petits groupes, en plénier), réguler les débats afin d'éviter les monopolisations de parole. À plusieurs reprises, des membres du groupe pilote, principalement les salariés d'AQUI Brie et de la chambre d'agriculture, sont mobilisés pour être animateurs. Des temps de préparation des animations et de débriefing sont organisés avant et après chaque atelier afin de partager les problèmes rencontrés et de proposer 
des améliorations. Ainsi, plusieurs reconnaissent avoir acquis des savoir-faire participatifs :

« Avant de commencer je n'étais pas forcément un grand praticien du dialogue territorial. Ça m'a rendu sans doute plus attentif aux discours des autres [...], ça participe à ma façon d'appréhender mon travail, je vois mieux les outils et comment on les utilise » (représentant AQUI Brie).

D'autres témoignent d'une appropriation de techniques d'animation qu'ils réinvestissent dans leur sphère professionnelle. C'est le cas du représentant de la chambre d'agriculture qui a réutilisé certains outils dans son animation du groupe agricole du Plan départemental de l'eau $(\mathrm{PDE})^{9}$ :

«Je me suis servi de tout ce que j'ai vu, ce qu'on a fait ensemble, des outils utilisés, de la manière d'animer les groupes, en petits groupes... je me suis inspiré de tout ça [...]. Ils ont tous apprécié le fait que j'utilise des outils qui n'étaient pas des choses habituelles en termes d'animation, en termes de travail. On n'avait jamais travaillé comme ça... Là, j'ai fait des sous-groupes, je les ai fait travailler sur des questions différentes, j'ai remis en commun... J'ai animé d'une façon complètement différente, avec une implication des gens».

Ce qui est appris est donc réinvesti et transféré dans d'autres espaces. C'est également ce que relate l'animatrice agricole d'AQUI Brie : « J'ai découvert une nouvelle façon de faire que j'ai essayé d'intégrer pour pouvoir la reproduire ». Cette expérience semble d'ailleurs avoir eu des effets plus profonds d'affirmation de son rôle:

«J'étais contente d'être là en tant qu'animatrice parce que ça renforçait mon identité au sein du territoire pour les agriculteurs [...]. Je pense que j'ai pris de l'assurance, justement parce que j'étais reconnue, que j'ai apporté des éléments qui étaient intéressants pour l'équipe, ça aide à se dire qu'on a une valeur ajoutée!».

L'analyse montre que la mise en tension des chercheurs et des acteurs est source d'apprentissages mutuels, ainsi que l'observe Jeanne Riaux à propos du travail interdisciplinaire: «Exacerbant les positions de chacun, il a provoqué des discussions, des oppositions, des incompréhensions, mais a permis ainsi de progresser vers un dialogue commun, d'initier des collaborations qui viennent transgresser des champs disciplinaires relativement étanches » (Riaux, 2013, p. 22). L'un des responsables du projet témoigne effectivement d'une expérience qui invite à «sortir de sa zone de confort habituelle » sans d'ailleurs toujours en voir, au départ, l'intérêt. Les nombreuses discussions ont pu être vécues comme des contraintes : les hésitations, les changements

\footnotetext{
9 Porté par le département de Seine-et-Marne, le PDE rassemble diverses institutions compétentes dans le domaine de l'eau pour engager des actions de protection de la ressource.
}

de cap obligent à repenser le cadrage et les outils, à les remettre plusieurs fois sur le métier avant de parvenir à une version collectivement partagée. Mais l'ensemble du groupe pilote en reconnaît l'intérêt à l'issue du processus.

\section{L'expérience participative: une transgression des frontières entre chercheurs, acteurs, monde agricole et monde de l'eau?}

L'examen du cœur de la démarche participative permet maintenant d'identifier les apprentissages chez les participants et d'analyser la manière dont ils contribuent à faire bouger les lignes qui divisent les parties prenantes du problème discuté. Il invite également à en repérer les limites, et notamment les asymétries d'apprentissages.

\section{Appropriation de nouveaux savoirs : la pédagogie du problème}

En imaginant d'autres manières de partager les savoirs (expertise des scientifiques, savoirs professionnels des agriculteurs, savoirs d'usage du territoire), la démarche contribue à une appropriation de nouveaux savoirs pour les acteurs locaux mobilisés. Nous identifions tout d'abord une amélioration des connaissances sur les zones tampons, notamment liée à une visite de la ZTHA expérimentale de Rampillon. Les entretiens conduits en début de processus avaient mis en évidence que la notion de «zone tampon » était principalement associée aux bandes enherbées. À l'issue de la démarche, les participants ouvrent la notion à une diversité d'objets, notamment les haies et zones humides, qu'ils n'identifiaient pas auparavant comme jouant un rôle de zone tampon:

«Au départ, j'étais néophyte... Je savais bien que l'eau de pluie traversait des cultures et ensuite elle allait aux rus et aux rivières, mais à part cela, je n'avais pas d'autres idées [...]. Là, $\mathrm{j}$ 'ai compris que plus on met de barrages à l'eau, plus les polluants sont retenus. C'est pour ça qu'on met de la végétation au bord des rus, qu'on laisse des espaces en herbe pour retenir les polluants avant que ça parte directement au ru et ensuite à la rivière» $(\text { ELU-EAU } 1)^{10}$.

${ }^{10}$ Les entretiens mobilisés ont été menés à l'issue de la démarche avec 15 participants : 6 agriculteurs (AGRI), 5 élus de collectivités dont certains sont agriculteurs (ELU-AGRI), et certains en charge de l'eau (ELU-EAU), 1 technicien de collectivité en charge de l'eau (TECH-EAU), 1 producteur privé d'eau potable (PROD-EAU), 2 acteurs des filières agricoles dont 1 représentante de la coopérative majoritaire du territoire (COOP) et 1 représentante de la filière biologique quasi inexistante sur le territoire (FIL-AB), et 1 association (ASSO). 
Plusieurs évoquent le fait que chacune de ces zones tampons correspond à des contextes et des enjeux spécifiques. Cela fait écho à une meilleure compréhension des mécanismes de circulation de l'eau, notamment la présence de gouffres karstiques accélérant le transfert de l'eau et des polluants de la surface vers les nappes souterraines :

«Je ne pensais pas que c'était aussi important [...]. Parce que là, c'est quand même pratiquement un million de mètres cubes qui s'engouffrent, sur une période courte. Donc, c'est vrai que sur 6 à 8 mois de l'année, c'est quand même impressionnant » (ELU-AGRI 1).

Les résultats sur l'efficacité de la ZTHA en termes de réduction des polluants sont également gardés en mémoire, en y intégrant les limites pointées par les chercheurs, notamment la faible efficacité pour certaines molécules chimiques:

«Il y a des produits qui rentrent et ne ressortent pratiquement pas, ils sont absorbés, travaillés, et d'autres qui rentrent, mais qui ressortent pratiquement à $80,90 \%$ [...]. Moi je pensais que l'on arrêtait à peu près tout à 30,40 , $50 \%$, alors que là, il y a des grosses différences entre matières actives » (AGRI 2).

Cet élément est identifié par certains agriculteurs comme un savoir susceptible d'avoir une utilité directe pour l'action :

«Ça m'a marqué parce qu'on peut l'exploiter. Et il faut l'exploiter [...]. Il faut utiliser les produits qui sont faciles à capter et puis éviter d'utiliser les produits qui traversent qu'on ne sait pas filtrer, qu'on ne sait pas retenir » (AGRI 2).

On note néanmoins que l'acquisition de nouveaux savoirs se fait dans la perspective d'un ajustement possible de leurs pratiques et non d'un changement radical: la prise de conscience de la variabilité des risques aurait aussi pu conduire à envisager l'arrêt de toute utilisation.

Les théories les plus récentes de l'apprentissage accordent une importance particulière à ce qu'elles nomment des "situations-problèmes », qui, confrontant des individus à une nécessité d'apprendre, favorisent l'acquisition de connaissances utiles à l'action (Fabre et Vellas, 2006). La perception d'un "problème sérieux et lourd de sens » (Rogers, 1998, p. 190) apparaît comme une condition de l'apprentissage, tout comme la conscience de pouvoir jouer un rôle dans la résolution de ce problème. Dans la reconnaissance du problème, la ZTHA expérimentale joue un rôle central, puisque c'est à travers les premières analyses des chercheurs que la présence de pesticides est rendue visible et que le lien avec les pratiques des agriculteurs est établi. Les pollutions diffuses, jusquelà invisibles, acquièrent un statut de «problème public ». Enfin, le cadre pédagogique proposé, notamment une visite de terrain commentée par les chercheurs qui montre la construction des savoirs scientifiques, constitue un autre élément favorisant ces apprentissages :

«Je trouve qu'on est trop dans des résultats de laboratoire alors que là, l'avantage, c'est qu'on a vraiment des résultats réels, enfin du terrain, avec aussi les inconvénients climatiques, la qualité des sols, tous les aléas [...]. Je trouve que là, on est vraiment dans la réalité de l'utilisation » (ELU-AGRI 1).

Cette visite de terrain joue un rôle capital par la présentation des instruments de mesure utilisés, l'explication des protocoles, les variations des résultats suivant les périodes de l'année, etc. Mais aussi par les aléas et les anecdotes venant parfois entraver les recherches -comme l'épisode des ragondins venus grignoter le matériel des chercheurs - et qui contribuent à construire une communauté d'intérêt autour des travaux des chercheurs, jusqu'à une appropriation par les agriculteurs qui n'hésiteront pas, ensuite, à contacter directement les chercheurs en cas de dysfonctionnement de la ZTHA.

\section{Changements de représentations: un apprentissage «à sens unique »}

Lors des ateliers animés avec l'outil Co-click'eau permettant de discuter de scénarios agronomiques, acteurs du monde agricole et du monde de l'eau sont amenés à partager différents savoirs et points de vue sur l'agriculture. Les ateliers sont l'occasion de poser des questions importantes pour des acteurs «non-initiés»: pourquoi tant de culture de blé ? À quoi est-il destiné ? Lors des débriefings et dans les entretiens, ces participants témoignent d'une meilleure compréhension du système agricole local :

«J'ai appris des choses sur les cultures, je ne savais rien $\mathrm{du}$ tout [...]. Je pensais qu'on ne faisait pas deux fois la même culture sur la même parcelle, mais en fait on peut. Et je pensais qu'après chaque récolte, on labourait, mais non, ça se fait pratiquement plus » (ELU-EAU 1).

Le fait d'avoir comme interlocuteurs des agriculteurs qui sont majoritairement sensibles au problème de qualité d'eau contribue à former une image plutôt positive de la profession, qui contraste avec celle, perçue à travers les médias, d'une profession particulièrement rétive au changement. Réciproquement, pour les agriculteurs, les ateliers sont vécus comme une occasion d'échanger dans des conditions favorables qui contrastent avec d'autres situations relatées comme conflictuelles: des tensions avec des habitants, des voisins qui se plaignent, des discussions parfois houleuses lors de repas de famille ou avec des amis, notamment depuis la médiatisation autour du glyphosate. L'un d'eux met en 
perspective ces difficultés avec la démarche participative proposée: «Là, c'était un peu l'occasion de discuter de ça, d'apprendre à mieux se connaître » (ELU-AGRI 3), reconnaissant l'importance de créer les conditions d'écoute, mais regrettant qu'elles soient rares.

On relève cependant des limites et des asymétries dans les apprentissages issus de cette démarche. Pour une partie des agriculteurs, les ateliers sont identifiés comme des fenêtres d'opportunité pour mieux communiquer, voire « redorer» leur image. Cela constitue parfois l'une des principales motivations de leur participation:

«Il y a de l'agri-bashing en ce moment, de façon assez importante à la télé, ça ne nous aide pas non plus. C'est pour ça qu'il faut qu'on aille plus à ces réunions pour pouvoir se faire comprendre et expliquer nos contraintes » (AGRI 14).

«C'est plus pour dire, nous, ce qu'on fait, et voir le ressenti des gens. Bon, ça, on le connaît un petit peu... Mais exposer ce qu'on fait, ce qu'on essaye d'améliorer [...], disons que nous, on y va surtout pour dire ce qu'on fait» (AGRI 13).

Et cela s'est observé lors des ateliers où les perceptions, attentes et contraintes du monde agricole se sont préférentiellement exprimées, rendant plus minoritaires celles des autres parties prenantes, notamment les collectivités en charge de l'eau potable. Cela conduit à infléchir la portée des apprentissages puisqu'on ne relève aucun témoignage d'agriculteurs indiquant une meilleure compréhension de ces acteurs et de leurs contraintes (notamment le problème grandissant de la dépollution de l'eau). Certains regrettent d'ailleurs cet apprentissage «à sens unique» :

«On s'est arrêtés à l'eau qui rentre dans la nappe et après on n'a pas parlé de l'eau qui sort [...]. Parce que ça coûte, les filtres à charbon, et c'est l'usine qui est chère au départ » (ELU-EAU 1).

Ce déséquilibre dans les traductions entre acteurs peut s'expliquer par le cadrage de la procédure (le coût de la production d'eau potable n'était, par exemple, pas représenté dans les différents outils mobilisés). Mais c'est aussi que des phénomènes de mise en retrait de points de vue environnementalistes face aux agriculteurs se sont opérés. L'envie - ou la nécessité - de construire un collectif, la crainte de voir les agriculteurs déserter les réunions ont pu conduire certains participants à restreindre leur expression. En entretien, un acteur associatif regrette que les paradigmes dominants soient restés ceux de la production agricole. Il évoque aussi l'attitude d'autocensure qu'il a adoptée à certains moments :

« Je n'ai pas trouvé qu'on a rappelé que l'eau c'est la vie. Donc à un moment il va falloir choisir, soit c'est la qualité de l'eau, soit c'est de la production [...]. Moi personnellement, j'ai toujours essayé d'être un peu sur la retenue [...]. J'étais plutôt en écoute. Sur le fait qu'à un moment, quelle est la priorité ? Si la priorité c'est qu'il y ait de l'eau consommable, il faut que l'eau soit consommable, quoi ! Donc à un moment, j'ai arrêté... Parce qu'après on se dit qu'on n'est peut-être pas à notre place pour dire ça, on peut peut-être rentrer dans un conflit...» (ASSO).

L'évitement des «moments sensibles» où les discussions montent en conflictualité est un phénomène observé dans d'autres dispositifs traitant d'eau et d'agriculture (Riegel, 2018; Seguin, 2020), mais aussi dans la vie démocratique plus généralement (Eliasoph, 1998). Cela n'est pas uniquement dû à l'animation du dialogue, mais aussi au fait que défendre une position face à des interlocuteurs en désaccord est une "pratique sociale risquée» (Duchesne et Haegel, 2004, p. 884) à laquelle on ne se livre pas spontanément. Mais cette observation appelle également une analyse réflexive sur nos positionnements en tant que porteurs du projet. Conscients des rapports de force inégaux entre acteurs environnementalistes et acteurs du monde agricole, aurait-il finalement été plus juste d'adopter un interventionnisme plus important et plus «orienté» dans les modalités d'animation? Autrement dit: «Faut-il ou non introduire une forme de discrimination positive pour corriger les asymétries de pouvoir au profit des plus défavorisés et/ou des "acteurs d'environnement" porteurs de la revendication écologique?» (Barbier et Larrue, 2011, p. 64). D'un autre côté, ce positionnement ne risque-t-il pas de porter atteinte à la légitimité du processus et de leurs porteurs? Cette interrogation est présente dans l'analyse de Barnaud et al. (2016) des différents types de positionnements chez les chercheurs et les praticiens de la participation. Dans notre cas, c'est certainement un des éléments explicatifs de cette transgression partielle des frontières.

\section{Vers de nouvelles formes d'organisations collectives?}

$\mathrm{Au}$ cours de cette démarche et à son issue, il est possible d'identifier des prémices de nouvelles formes d'organisations collectives. Même s'il est encore trop tôt pour parler de véritables apprentissages organisationnels, plusieurs indices laissent entrevoir une inflexion dans les manières de penser l'action collective locale sur les enjeux d'eau et d'agriculture. Le premier concerne l'attrait pour les formes participatives proposées. Les participants font une évaluation positive des différents outils et méthodes d'animation par contraste avec des situations plus classiques de réunion. Les échanges en petits groupes sont identifiés comme des moments utiles et efficaces. C'est également le caractère interactif qui est apprécié : 
«C'est efficace dans le sens où chacun peut s'exprimer, je trouve. On est plus acteur que consommateur, sinon on écoute la personne parler et on consomme. Là, on participe, c'est le terme. C'est plus vivant, c'est plus motivant. On rentre dans le jeu [...]. Là, on peut poser des questions au fur et à mesure du débat. On est plus réactif aussi » (ELU-AGRI 2).

«Je pense que ça contribue aussi au fait que chacun puisse s'exprimer, que ce ne soit pas ceux qui sont les plus sûrs d'eux ou ceux qui ont la voix qui porte [...] qui s'expriment, et au final on croit que c'est l'avis de tout le monde alors qu'en fait, non. Donc ça, je trouve ça intéressant, et puis du coup, chacun est plus impliqué et est plus acteur » (FIL-AB).

C'est une expérience jugée utile pour repenser d'autres situations rencontrées dans le cadre professionnel:

«Ça permet de se mettre à la place des autres acteurs. Disons qu'après, quand on se retrouve dans une vraie situation, de pouvoir un peu éviter les postures toutes faites et être un peu plus dans l'écoute des autres, ça peut être utile, oui, pour mieux dialoguer, pour mieux vraiment se mettre dans sa peau » (FIL-AB).

Le second indice concerne la manière de construire les savoirs sur ces enjeux. La visite de terrain est relatée comme un moment fort de l'expérience et lors de l'atelier final est exprimée l'envie de poursuivre ce meilleur partage des savoirs avec les scientifiques. Lorsque ceuxci font part des expérimentations à venir, plusieurs agriculteurs se disent intéressés pour les suivre. On peut y voir l'amorce d'un renouvellement des manières de concevoir la production et le partage des savoirs avec les agriculteurs, une ouverture des travaux scientifiques vers une forme «semi-confinée» (Callon et al., 2001).

Le troisième indice est l'expression d'une volonté de s'affranchir des cadres institutionnels habituels contraignant le dialogue et l'action. Une représentante d'un producteur d'eau potable, amenée à travailler avec la profession agricole dans le cadre de la protection de captages, évoque ainsi le contraste entre cette démarche et des expériences antérieures de travail avec des interlocuteurs institutionnels, notamment la chambre d'agriculture. Les ateliers ont mis en évidence les différences de discours et de postures qui existent entre représentants de la profession et agriculteurs n'ayant pas de fonction de représentation:

«De voir qu'il y avait déjà des agriculteurs partant pour faire changer les choses, ça fait plaisir parce que des fois, justement, en écoutant le discours de la chambre c'est «Oh non, non, on ne peut rien faire!». Alors que si, quand on les rencontre, il y en a qui sont prêts. Donc ça, par contre, c'est vraiment ce que m'ont appris ces ateliers [...]. C'est pour ça qu'il ne faut pas qu'il y ait d'intermédiaires » (PROD-EAU).

Cet apprentissage a des conséquences sur les pratiques puisque cette professionnelle de l'eau potable reconnaît avoir modifié depuis sa stratégie de mobilisation des agriculteurs :

«Ça nous a permis de remettre en question la manière dont on travaillait dans ce plan d'action-là et de se dire : il faut trouver un moyen pour réussir à accrocher les agriculteurs, à les intéresser. Je pense que ça nous a aidés de ce point de vue-là » (PROD-EAU).

Enfin, le fait que ce dialogue prenne place dans le cadre d'un projet de recherche sans obligation d'aboutir à un plan d'action à valeur réglementaire a été reconnu comme favorisant un dialogue sans grande animosité.

Dernier indice, la naissance de solidarités de territoire entre acteurs agricoles et non agricoles. Cela contraste avec d'autres formes de solidarité, notamment de corps professionnel, très influentes dans le monde agricole. Lors du dernier atelier, les participants évoquent plusieurs pistes : l'identification de lieux pertinents pour l'installation de zones tampons via une cartographie collective, de nouveaux modes d'organisation pour l'aménagement et l'entretien des éléments du paysage, «un système de gestion en commun» ou de nouvelles formes partenariales visant à «recréer des communs » à inventer sur un territoire marqué par l'emprise des propriétés privées. Un agriculteur évoque la possibilité de réveiller une ancienne forme d'association syndicale que sont les associations foncières de remembrement, mais cette fois-ci dans le but de restaurer les éléments du paysage ayant disparu. Enfin, plusieurs propositions sont faites en direction du monde agricole: organiser des visites d'exploitations pour faire connaître les pratiques respectueuses de l'environnement, développer de nouvelles formes de commercialisation et de consommation des produits agricoles, plus locales, plus créatrices d'emploi, et s'inscrivant dans des logiques collaboratives comme les AMAP. On voit ici comment, d'un enjeu initialement centré sur une innovation technique permettant de réduire les transferts de polluants sans impliquer nécessairement de changements de système agricole, s'ouvre une réflexion sur les modes d'organisation économique et sociale du territoire. Ces différents éléments s'inscrivent dans une volonté de collaboration plus étroite entre acteurs locaux issus de mondes cloisonnés, mais ayant identifié de nouvelles alliances possibles.

\section{Conclusion}

L'analyse a conduit à identifier deux formes de relations entre cadrage de la participation et apprentissages suscités chez les différents acteurs. D'une part, la co-ingénierie de la participation au sein du groupe projet est génératrice d'apprentissages venant questionner et dépasser le cadre initial du projet. Prenant acte de 
l'étroitesse de la commande de départ qui relève d'une construction de l'acceptabilité sociale d'une innovation technique, le groupe transforme et élargit son objet et ses objectifs chemin faisant. D'autre part, au cours de l'expérience participative, les apprentissages sont réels mais contraints par le cadre. Ce cadre est à la fois celui donné par les porteurs du projet, mais également celui intégré par les participants pris dans des rapports de pouvoir inégaux. L'expérience a certes permis de faire un premier pas sur ce territoire façonné et dominé par le monde agricole, mais il reste un chemin important à réaliser pour rendre entièrement discutable l'enjeu de la qualité de l'eau. La participation ne résout pas toutes les asymétries entre parties prenantes des enjeux environnementaux (Barnaud, 2013).

Néanmoins, même s'ils appellent à une analyse nuancée, les apprentissages relevés au cours de cette expérience sont intéressants. Les savoirs et savoir-faire partagés et acquis par certains, invisibles s'ils ne font pas l'objet d'une évaluation spécifique, peuvent mener à des changements dans les manières de construire les connaissances et de s'organiser collectivement, voire, à plus long terme, dans les processus de prise de décision incluant un groupe d'acteurs plus large. Ces effets indirects et à plus long terme ne sont pas ici pris en compte mais pourront faire l'objet d'une évaluation ultérieure. Il serait notamment utile de voir si ces apprentissages sont susceptibles de se traduire ensuite dans des habitudes, des stratégies, voire des routines collectives plus pérennes et indépendantes des individus.

\section{Remerciements}

Ce travail a été mené grâce au financement des programmes PSDR (Pour et sur le développement régional) de la région Île-de-France et du PIREN-Seine (Programme interdisciplinaire de recherche sur l'eau et l'environnement du bassin de la Seine). Les auteurs remercient l'ensemble des partenaires scientifiques et institutionnels ainsi que les acteurs du territoire de l'Ancœur qui ont pris part au projet Brie'eau.

\section{Références}

Barataud F., Arrighi A., Durpoix A., 2015. Mettre cartes sur table et parler de son territoire de l'eau: un (en)jeu pour les acteurs?, VertigO - La revue électronique en sciences de l'environnement, 15, 3, https://doi.org/10.4000/ver tigo. 16766 .

Barataud F., Hellec F., Levain A., Petit S., 2017. Quand les recherches en sciences sociales s'invitent dans la gestion de l'eau, Natures Sciences Sociétés, 26, 4, 395-406, https://doi. org/10.1051/nss/2018054.
Barbier R., Larrue C., 2011. Démocratie environnementale et territoires: un bilan d'étape, Participations, 1, 67-104, https://doi.org/10.3917/parti.001.0067.

Barnaud C., 2013. La participation, une légitimité en question, Natures Sciences Sociétés, 21, 1, 24-34, https://doi.org/ $10.1051 / \mathrm{nss} / 2013062$.

Barnaud C., D’Aquino P., Daré W., Mathevet R., 2016. Dispositifs participatifs et asymétries de pouvoir : expliciter et interroger les positionnements, Participations, 16, 3, 137-166, https://doi.org/10.3917/parti.016.0137.

Barnett C.K., 2001. Rethinking organizational learning theories: a review and synthesis of the primary literature, Academy of Management review, non publié.

Barthe Y., 2002. Rendre discutable. Le traitement politique d'un héritage technologique, Politix, 57, 57-78, https://doi. org/10.3406/polix.2002.1207.

Billaud J.-P., 2003. De l'objet de l'interdisciplinarité à l'interdisciplinarité autour des objets, Natures Sciences Sociétés, 11, 1, 29-36, www.nss-journal.org/fr/articles/nss/ pdf/2003/01/nss20031101p29.pdf.

Blondiaux L., Fourniau J.-M, 2011. Un bilan des recherches sur la participation du public en démocratie : beaucoup de bruit pour rien?, Participations, 1, 8-35, https://doi.org/ 10.3917/parti.001.0008.

Callon M., Lascoumes P., Barthe Y., 2001. Agir dans un monde incertain. Essai sur la démocratie technique, Paris, Seuil.

Crookall D., 2014. Engaging (in) gameplay and (in) debriefing, Simulation \& Gaming, 45, 4-5, 416-427, https://doi.org/ 10.1177/1046878114559879.

Daniell K.A., White I., Ferrand N., Ribarova I.S., Coad P., Rougier J.-E., Hare M., Jones N.A., Popova A., Rollin D., Perez P., Burn S., 2010. Co-engineering participatory water management processes: theory and insights from Australian and Bulgarian interventions, Ecology and Society, 15, 4, www.ecologyandsociety.org/vol15/iss4/art11/.

Daré W., Van Passen A., Ducrot R., Mathevet R., Queste J., Trébuil G., Brabaud C., Lagabrielle E., 2010. Apprentissage des interdépendances et des dynamiques, in Étienne $\mathrm{M}$. (Ed.), La modélisation d'accompagnement. Une démarche participative en appui au développement durable, Versailles, Quæ, 223-250.

Duchesne S., Haegel F., 2004. La politisation des discussions, au croisement des logiques de spécialisation et de conflictualisation, Revue française de science politique, 54, 6, 877-909, https://doi.org/10.3917/rfsp.546.0877.

Eliasoph N., 1998. Avoiding politics. How Americans produce apathy in everyday life, Cambridge, Cambridge University Press.

Étienne M., Du Toit D.R., Pollard S., 2011. ARDI: a coconstruction method for participatory modeling in natural resources management, Ecology and Society, 16, 1, 44, www.ecologyandsociety.org/vol16/iss1/art44/.

Fabre M., Vellas E. (Eds), 2006. Situations de formation et problématisation, Bruxelles, De Boeck.

Fourniau J.-M., 2007. L'expérience démocratique des «citoyens en tant que riverains dans les conflits d'aménagement, Revue européenne des sciences sociales, XLV-136, 149-179, https://doi.org/10.4000/ress.95. 
Gardesse C., 2011. L'évaluation de dispositifs participatifs par l'analyse de leurs effets : le cas de la "concertation" pour le projet de réaménagement des Halles de Paris, 2002-2010. Communication à la $2^{e}$ journée doctorale sur la participation du public et la démocratie participative, École des hautes études en sciences sociales, 18 octobre, Paris, www.participation-et-democratie.fr/system/files/ate lier_4-4_camille_gardesse.pdf.

Genieys W., Hassenteufel P., 2012. Qui gouverne les politiques publiques? Par-delà la sociologie des élites, Gouvernement et action publique, 2, 1, 89-115, https://doi.org/10.3917/ gap.122.0089.

Gisclard M., Chantre E., Cerf M., Guichard L., 2015. Coclick'eau: une démarche d'intermédiation pour la construction d'une action collective locale?, Natures Sciences Sociétés, 23, 1, 3-13, https://doi.org/10.1051/nss/2015003.

Goffman E., 1974. Frame analysis: an essay on the organization of experience, New York, Harper \& Row.

Jollivet M., Legay J.-M., 2005. Canevas pour une réflexion sur une interdisciplinarité entre sciences de la nature et sciences sociales, Natures Sciences Sociétés, 13, 2, 184-188, https:// doi.org/10.1051/nss:2005030.

Lascoumes P., 2010 [1 ${ }^{\text {re }}$ éd. 2004]. Traduction, in Boussaguet L., Jacquot S., Ravinet P. (Eds), Dictionnaire des politiques publiques, Paris, Presses de Sciences Po, 632-640.

Nez H., 2011. Nature et légitimités des savoirs citoyens dans l'urbanisme participatif. Une enquête ethnographique à Paris, Sociologie, 2, 4, 387-404, https://doi.org/10.3917/ socio.024.0387.

Raymond R., 2017. Transition énergétique et transdisciplinarité, Natures Sciences Sociétés, 25, S42-S44, https://doi. org/10.1051/nss/2017030.

Reed M.S., 2008. Stakeholder participation for environmental management: a literature review, Biological Conservation, 141, 10, 2417-2431, https://doi.org/10.1016/j.bio con.2008.07.014.

Reed M.S., Evely A.C., Cundill G., Fazey I., Glass J., Laing A., Newig J., Parrish B., Prell C., Raymond C., Stringer L. C., 2010. What is social learning?, Ecology and Society, 15, 4, http://www.ecologyandsociety.org/vol15/iss4/resp1/.

Riaux J., 2013. Engager la construction d'un regard sociohydrologique: des archives catalyseurs de l'interdisciplinarité, Natures Sciences Sociétés, 21, 1, 15-23, https:// doi.org/10.1051/nss/2013061.

Riegel J., 2018. Le dialogue territorial au risque de l'écologie? Traces et effets d'une concertation entre aménagements hydrauliques et restauration écologique, Participations, 20, 1, 173-198, https://doi.org/10.3917/parti.020.0171.
Rogers C.R., 1998 [1 $1^{\mathrm{re}}$ éd. 1966]. Le développement de la personne, Paris, Dunod.

Rui S., 2004. La démocratie en débat. Les citoyens face à l'action publique, Paris, Armand Colin.

Sabatier P.A., Jenkins-Smith H.C., 1993. Policy change and learning: an advocacy coalition approach, Boulder, Westview Press.

Seguin L., 2020. Apprentissages de la citoyenneté. Expériences démocratiques et environnement, Paris, Éditions de la Maison des sciences de l'homme.

Seguin L., Barataud F., 2021. Quand la trajectoire d'objets techniques liés à la gestion des eaux agricoles révèle des valeurs attachées à la nature, VertigO-la revue électronique en sciences de l'environnement, 21 , 1, https://doi.org/ 10.4000/vertigo.31870.

Seguin L., Birmant F., Letournel G., Bonifazi M., Barataud F., Arrighi A., Guichard L., Bouarfa S., Roger L., Royer L., Hureau D., Rougier J.-E., Melion-Delage R., Bontoux C., Berthomé B., Tournebize J., 2018. Projet BRIE'EAU : une démarche participative pour repenser ensemble un territoire de grandes cultures, Agronomie, Environnement \& Sociétés, 8, 2, 157-169, https://agronomie.asso.fr/aes-8-2-21.

Simard L., Fourniau J.-M., 2007. Ce que débattre nous apprend. Éléments pour une évaluation des apprentissages liés au débat public, in Revel M., Blatrix C., Blondiaux L., Fourniau J.-M., Hériard Dubreuil B., Lefebvre R. (Eds), Le débat public: une expérience française de démocratie participative, Paris, La Découverte, 318-331.

Soulard C.T., Compagnone C., Lémery B., 2007. La recherche en partenariat: entre fiction et friction, Natures Sciences Sociétés, 15, 1, 13-22, https://doi.org/10.1051/nss:2007019.

Talpin J., 2011. Schools of democracy. How ordinary citizens (sometimes) become competent in participatory budgeting institutions, Colchester, ECPR Press.

Tournebize J., Gramaglia C., Birmant F., Bouarfa S., Chaumont C., Vincent B., 2012. Co-design of constructed wetlands to mitigate pesticide pollution in a drained catch-basin: a solution to improve groundwater quality, Irrigation and Drainage, 61, Suppl. 1, 75-86, https://doi.org/10.1002/ird.1655.

Tournebize J., Chaumont C., Mander Ü., 2017. Implications for constructed wetlands to mitigate nitrate and pesticide pollution in agricultural drained watersheds, Ecological Engineering, 103, Part B, 415-425, https://doi.org/10.1016/ j.ecoleng.2016.02.014.

Webler T., Kastenholz H., Renn O., 1995. Public participation in impact assessment: a social learning perspective, Environmental Impact Assessment Review, 15, 5, 443463, https://doi.org/10.1016/0195-9255(95)00043-E.

Citation de l'article: Seguin L., Barataud F., Guichard L., Bonifazi M., Souchère V., Bouarfa S., Tournebize J. La participation comme objet intermédiaire d'apprentissages : leçons d'une démarche participative sur les pollutions diffuses agricoles. Nat. Sci. Soc. 29, 3, 299-311. 